The Joint $28^{\text {th }}$ ICFA Advanced Beam Dynamics and Advanced \& Novel Accelerators Workshop

\title{
Quantum Aspects OF BEAM PHYSICS
}

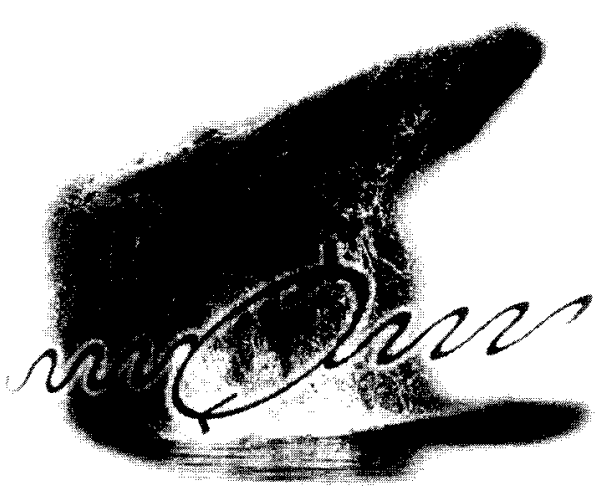




\section{Also published by World Scientific}

Quantum Aspects of Beam Physics

Advanced ICFA Beam Dynamics Workshop on

Edited by Pisin Chen

ISBN: 981-02-3551-8

Quantum Aspects of Beam Physics

$18^{\text {th }}$ Advanced ICFA Beam Dynamics Workshop on

Edited by Pisin Chen

ISBN: 981-02-4950-0 
The Joint $28^{\text {th }}$ ICFA Advanced Beam Dynamics and Advanced \& Novel Accelerators Workshop

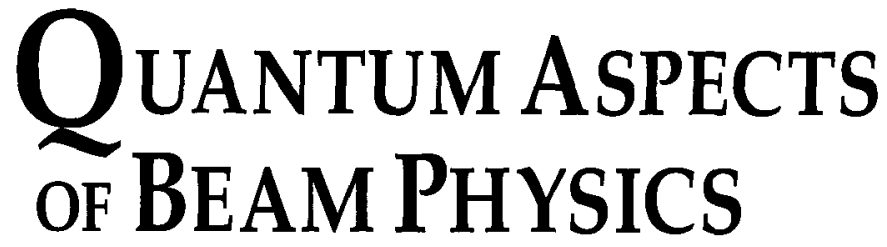

Hiroshima, Japan $\quad 7-11$, January 2003

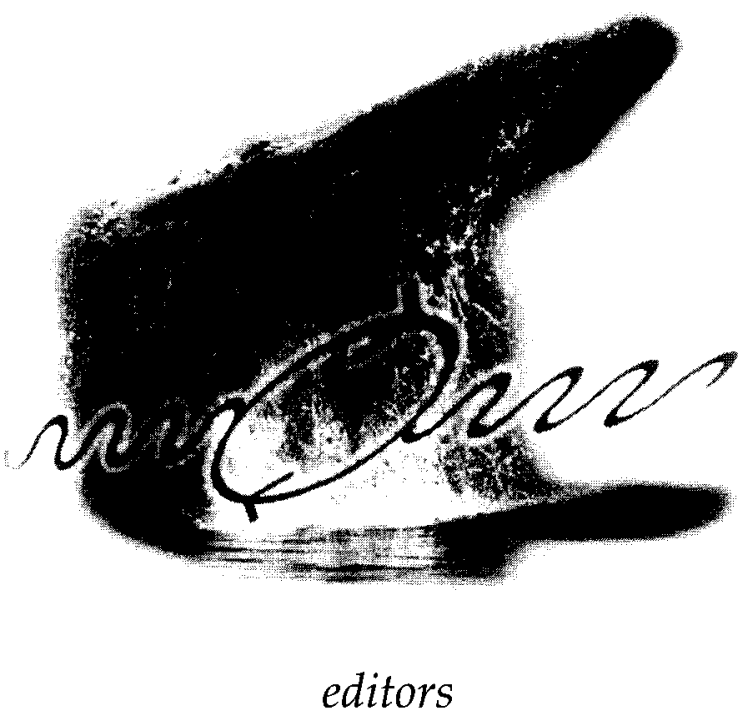

Pisin Chen \& Kevin Reil Stanford Linear Accelerator Center, USA 


\section{Published by}

World Scientific Publishing Co. Pte. Ltd.

5 Toh Tuck Link, Singapore 596224

USA office: 27 Warren Street, Suite 401-402, Hackensack, NJ 07601

UK office: 57 Shelton Street, Covent Garden, London WC2H 9HE

\section{British Library Cataloguing-in-Publication Data}

A catalogue record for this book is available from the British Library.

\section{QUANTUM ASPECTS OF BEAM PHYSICS 2003}

Proceedings of the 28th ICFA Advanced Beam Dynamics and Advanced \& Novel Accelerators Workshop

Copyright $(2004$ by World Scientific Publishing Co. Pte. Ltd.

All rights reserved. This book, or parts thereof, may not be reproduced in any form or by any means, electronic or mechanical, including photocopying, recording or any information storage and retrieval system now known or to be invented, without written permission from the Publisher.

For photocopying of material in this volume, please pay a copying fee through the Copyright Clearance Center, Inc., 222 Rosewood Drive, Danvers, MA 01923, USA. In this case permission to photocopy is not required from the publisher.

ISBN 981-256-069-6 


\section{PREFACE}

The Joint $28^{\text {th }}$ ICFA (International Committee for Future Accelerators) Advanced Beam Dynamics and Advanced and Novel Accelerators Workshop on "QUANTUM ASPECTS OF BEAM PHYSICS and Other Critical Issues of Beams in Physics and Astrophysics", was held on January 7-11, 2003 , in Hiroshima, Japan. This was the third in the QABP workshop series. The first QABP workshop was launched in January 1998, in Monterey, California, and the second was held in October 2000, in Capri, Italy. Over the past five years, this workshop series has passed its torch around the world, from the U.S. to Europe, and this time to Japan in Asia. Following the footsteps of the first two workshops, this one in Hiroshima was again a tremendous success.

The frontier of beam research points to increasingly higher energy, greater brightness and lower emittance beams with ever-increasing particle species. These demands have triggered a rapidly growing number of beam phenomena that involve quantum effects. With the significant advancement of laser and accelerator technologies, there is also a growing interest in using high energy, high intensity particle and photon beams for laboratory astrophysics investigations, as well as the application of beam physics expertise to astrophysics studies. It has therefore become a tradition that this workshop series attracted a broad spectrum of experts from beam physics, astrophysics, cosmology, particle physics, condensed matter physics, nuclear physics, atomic physics, and laser science, to explore a common frontier where their individual expertise and interests overlapped.

About 70 physicists from China, Denmark, Georgia, Germany, India, Italy, Japan, Korea, Mexico, Mongolia, Russia, Taiwan, Ukraine, U.K., and U.S., had participated in the Hiroshima workshop. Unlike the first two workshops, the Hiroshima meeting was conducted in the symposium style. Other than one poster session, all the meetings were plenary. The Program Committee thought that the symposium style would allow participants to listen to all interesting talks. The concern, however, was the loss of opportunity for free exchange of ideas under such a format. To prevent such a drawback, the workshop had set-aside a block of time for roundtable discussion at the end of each topical session. This was found to be a very welcome arrangement. In the end the advantages of both workshop and symposium styles appeared to have preserved. The exciting scientific program is now documented in this volume. 
The social program of the Hiroshima workshop was also wonderfully organized by the Chairman of the Organizing Committee, Atsushi Ogata. Following the tradition of the QABP workshop series, which seeks to actively integrate science and culture, there was again an opening night music concert at the workshop, performed by concert marimbist, Ms. Hiroko Tsuji. Her repertoire extends from classical music of J.S. Bach to contemporary American and Japanese pieces. The excursion to the famous and sacred Miyajima Island in the Hiroshima Bay was most memorable. Hiroshima is home to prestigious Sake breweries. The post-workshop visit to one of the famous breweries was very educational. Many foreign workshop participants also took extra time to visit the atomic bomb site and the Memorial Piece Museum in Hiroshima.

None of these wonderful arrangements were possible without the dedication and outstanding organizational skill of Prof. Atsushi Ogata and his staff of the Hiroshima University. On behalf of all conference participants, I wish to express our sincere gratitude to his effort. With the successful ending of the QABP03 Hiroshima meeting, the trilogy of workshops on quantum beam physics has by now come around the world in a full circle.

Six years ago, I designed the QABP workshop logo (a jumping whale overlaps with a vacuum-polarization Feynman diagram) under the inspiration that there was an interesting analogy between emerging whales from the sea and vacuum fluctuations in the Dirac "sea", and with the conviction that the ultimate understanding of the physical vacuum was one of the last frontiers in physics. In the interim, we saw the emergence of the notion of dark energy in cosmology, whose nature is beyond anyone's comprehension. Although it is unclear which water will this QABP whale swim to next, it is clear that the subject will continue to be in the forefront of our pursuit.

\author{
Pisin Chen \\ Stanford, California
}




\section{CONTENTS}

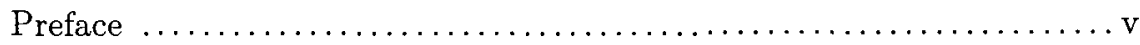

P. Chen

I. Quantum Fluctuations and Methodologies in Beam Physics

On Coherent Radiation in Electron-Positron Colliders ................ 3

V. N. Baier, V. M. Katkov

Quantum Mechanics of Dirac Particle Beam Transport Through

Optical Elements with Straight and Curved Axes

R. Jaganathan

Pattern Formation in Wigner-Like Equations via Multiresolution .....22

A. N. Fedorava, M. G. Zeitlin

The Halo Formation in Charged Particle Beams: A

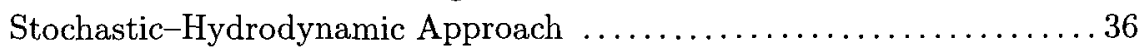

N. C. Petroni, S. De Martino, S. De Siena, F. Illuminati

\section{Photon-Electron Interaction in Beam Production, Cooling and Monitoring}

Compton Scattering and its Applications: The Pleiades

Femtosecond X-Ray Source at LLNL ........................ 49

F. V. Hartemann, W. J. Brown, S. G. Anderson, C. P. J. Barty,

S. M. Betts, R. Booth, J. K. Crane, R. R. Cross, D. N. Fittinghoff, D. J. Gibson, J. Kuba, B. Rupp, A. M. Tremaine, P. T. Springer

Infrared Synchrotron Radiation in Accelerator Experiments

A. A. Maltsev

Infrared Coordinate Detector for the Measurement of Angular

Divergence in Synchrotron Radiation

A. A. Maltsev, M. A. Maltsev, M. V. Mastova 
Study of Laser Compton Scattering in a Plasma Channel 80

T. Kumita, Y. Kamiya, I. Ben-Zvi, K. Kusche, I. V. Pogorelsky, V. Yakimenko, D. P. Siddons, T. Hirose, S. Kashiwagi, M. Washio, D. Cline, F. Zhou, D. Kaganovich, A. Zigler

Irreversible Process in a Photon Gas 86

N. L. Tsintsadze, K. Nishikawa, Y. Kishimoto, N. A. Papuashvili, L. N. Tsintsadze

Production of a Short-Bunch Polarized Positron Beam and Design of a Positron Polarimeter ................................ 95

M. Fukuda, A. Ohashi, T. Hirose, M. Washio, I. Yamazaki,

Y. Kurihara, M. Nomura, T. Okugi, T. Omori, J. Urakawa

X-Ray Spectrometer for Observation of Nonlinear Compton

Scattering

Y. Kamiya, T. Kumita, D. P. Siddons, I. Ben-Zvi, C. Liu, A. T. Macrander, T. Hirose, M. Washio, T. Omori, J. Urakawa, K. Yokoya

Application of Optical Diffraction Radiation to a Non-Invasive Low-Emittance High-Brightness Beam Diagnostics

P. Karataev, R. Hamatsu, T. Muto, S. Araki, H. Hayano,

J. Urakawa, G. Naumenko, A. Potylitsyn, T. Hirose

Generation of High Quality Electron Beam by Laser-Plasma Interaction

H. Kotaki, S. Masuda, M. Kando, S. Kondo, S. Kanazawa,

T. Homma, K. Nakajima

Polarization of Final Photons in Nonlinear Compton Scattering for Linearly Polarized Laser

L. Dongguo, R. Hamatsu, T. Sumiyoshi, K. Yokoya, T. Hirose

Is it Possible to Obtain Polarized Positrons During Multiple Compton Backscattering Process?

\section{A. P. Potylitsyn}

1D PIC Simulation of Plasma Cathode

S. Masuda, M. Kando, H. Kotaki, S. Kondo, S. Kanazawa,

T. Honma, I. V. Smetanin, K. Nakajima 


\section{Beam Phenomena under Strong Fields}

Boiling the Vacuum with an X-Ray Free Electron Laser

A. Ringwald

Unruh Effect as Particular Frenet-Serret Vacuum Radiation and

Detection Proposals

H. C. Rosu

Quantum Excitation-Free Radiation Emission Including Multiple

Scattering

U. I. Uggerh $\varnothing j$

Flying Mirrors: Relativistic Plasma Wake Caustic Light

Intensification

T. Zh. Esirkepov, S. V. Bulanov, T. Tajima

Quantum Theory of Laser-Beam Compton Interaction and Stimulated

Electron-Positron Annihilation in a Strong Field

I. V. Smetanin, K. Nakajima

Interaction of Charged Particles with Ultra Strong

Electromagnetic Waves in the Radiation Dominant Regime ........ 204

S. V. Bulanov, T. Zh. Esirkepov, J. Koga, T. Tajima

Electron-Positron Pair-Beam Production and Acceleration in Ultra-Strong Laser-Plasma Interactions . ..................... 218

K. Nakajima

Quantum Aspects of Super-Strong Field Interactions

H. Takahashi

\section{High Energy and Laboratory Astrophysics}

A Crystal-Based Matter Wave Interferometric

Gravitational-Wave Observatory

R. Y. Chiao, A. D. Speliotopoulos 
Gravitational-Wave Induced Effects in Storage Rings Beam Dynamics

D. Dong, C.-G. Huang

Cosmological Final Focus Systems

J. Irwin, M. Shmakova

Spontaneous Gravitational Instability of Star Distribution in a

Nonrotating Galaxy

A. Chao

The Search for Quantum Gravity Using Matter Interferometers

287

R. Bingham

Electron-Positron-Photon Plasma Around a Collapsing Star

R. Ruffini, L. Vitagliano, S.-S. Xue

Plasma Expansion in the Geometry of a Collapsing Star

R. Ruffini, L. Vitagliano, S.-S. Xue

Generalized Uncertainty Principle and Dark Matter

P. Chen

CP Violation of the Early Universe and the Mass Scale of Heavy

Majorana Neutrinos

T. Morozumi

Ultra-High Energy Cosmic Rays and Violation of Lorentz Invariance Induced by External Field

H. Sato

Bridging Particle Astrophysics and Cosmology via Ultra High Energy Cosmic Rays

W-Y.P. Hwang

The Lamb Shift and Ultra High Energy Cosmic Rays 353

S.-S. Xue

Ultra High Energy Cosmic Rays and the Fluorescence in Air Showers (FLASH) Experiment at SLAC 359 J. N. Matthews 
An Overview of Astrophysical Accelerators

F. C. Jones

Cosmic Plasma Wakefield Acceleration

P. Chen, T. Tajima, Y. Takahashi

Accelerator Experiments for Astrophysics

J. S. T. $N g$

V. Critical Issues in Linear Colliders, Plasma Accelerators, and Ultra Cold Beams

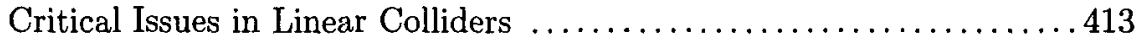

V. Telnov

Recent Progress on Photon Colliders

T. Takahashi

Beam-Size Effect in Bremsstrahlung

V. N. Baier, V. M. Katkov

Critical Issues for Vacuum Laser Acceleration

E. R. Colby

Critical Issues in Plasma Accelerator

M. Uesaka, T. Hosokai

Proposed Laser Driven Photonic Band Gap Accelerator

J. H. Han, I. S. Ko

Use of Macroscopic Solid Structure in Accelerators

A. Ogata, N. Saito, T. Ishimaru

Observations on Bunched Crystalline Beams

U. Schramm, M. Bussmann, T. Schätz, D. Habs

Approach to Ultra Cold Beam at LSR, Kyoto University ...........500

A. Noda, H. Fadil, M. Ikegami, Y. Iwashita, T. Shirai,

H. Tongu, K. Noda, S. Shibiya, T. Takeuchi 


\section{Appendices}

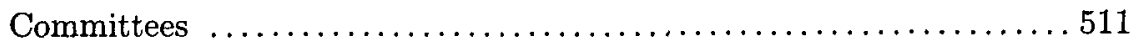

List of Participants .................................. 512

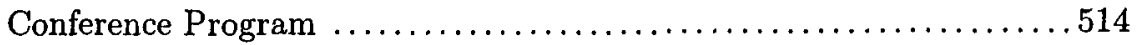

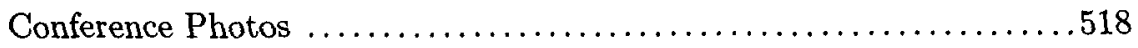

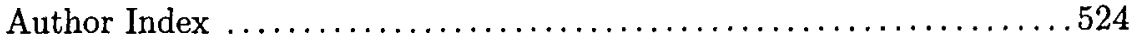

